\title{
Childhood sexual abuse: disclosure and mandatory reporting - ethical and clinical implications
}

\author{
William Burbridge-James
}

\begin{abstract}
SUMMARY
The widespread nature of child sexual abuse is increasingly recognised and in the public domain following high-profile cases. Children and vulnerable people need protecting from such abuse. At the heart of the dilemma facing clinicians is the need to feel supported by the legislative framework to maintain confidentiality and use their clinical discretion in the best interests of their patients, ensuring that they receive the help they need, but also protecting others at risk. There are compelling arguments both for and against strengthening legislation to improve child protection.
\end{abstract}

\section{LEARNING OBJECTIVES}

- To understand the context for the potential introduction of mandatory reporting.

- To understand the arguments for and against mandatory reporting of childhood, including historic, sexual abuse.

- To reflect on the implications of mandatory reporting of childhood, including historic, sexual abuse for readers clinical practice.

\section{DECLARATION OF INTEREST}

None.

All health professionals, but particularly psychiatrists of all specialties, encounter reported childhood sexual abuse in their clinical work and its consequent, often devastating, impact on the lives of their patients.

The presence of childhood sexual abuse in the aetiology of psychiatric disorders is well recognised across multiple diagnostic domains, including psychosis (Chen 2010), personality disorder (Varese 2012), complex post-traumatic stress disorder, depression, anxiety, substance misuse and psychosomatic disorders. The Adverse Childhood Experiences (ACE) Study (Zanarini 1997) illustrates the effects of ACEs, which include childhood sexual abuse (reported by $21 \%$ of the cohort), on subsequent physical and mental health, with the development of chronic health conditions and shortened lifespans (Felitti 1998).
Interventions that might reduce the high incidence of child sexual abuse $-11 \%$ of women and $3 \%$ of men report being sexually assaulted as minors (Flatley 2016) - and are therefore preventive in the development of future morbidity should be viewed as potentially positive.

\section{The context for the case for mandatory reporting}

The revelations relating to Operation Yewtree in 2012, which investigated the abuse carried out by Jimmy Savile and other high-profile celebrities, brought to light the prolific scale and time span of Savile's offending, with 450 victims coming forward to report abuse that ranged over a period of 54 years. The extent of Savile's offending is illustrated in a joint Metropolitan Police Service and National Society for the Prevention of Cruelty to Children (NSPCC) report into allegations of sexual abuse made against him (Gray 2013).

The NSPCC viewed the Savile and other cases as exposing weaknesses in the protection of children in environments such as hospitals, schools, detention facilities and, recently, football clubs, where in many instances individuals were aware of what was happening but failed to take effective action. It convened a round-table discussion to explore the case for mandatory reporting, stating that 'there is compelling evidence that existing arrangements have not been sufficient to protect children' (NSPCC 2014: p. 11).

Under mandatory reporting, specific groups of professionals would be placed under a legal duty to report suspected cases of child abuse and neglect to proper authorities. These groups are referred to as 'mandated reporters'. Failure to report reasonably held concerns would lead to criminal sanctions.

\section{'Wilful neglect' and the culture of denial}

In 2015, following publication of reviews into child sexual exploitation that revealed social work failings in Rotherham, Rochdale and Derby, the then Prime Minister David Cameron outlined plans for a government consultation on extending the criminal
CLINICAL REFLECTION
William Burbridge-James is a consultant psychiatrist in medical psychotherapy in Southend-on-Sea, Essex, and Chair of the Specialty Advisory Committee of the Faculty of Medical Psychotherapy at the Royal College of Psychiatrists.

Correspondence Dr William Burbridge-James, The Taylor Centre, Warrior House, 42-82 Southchurch Road, Southend-on-Sea SS1 2LZ, UK. Email: william.burbridge-james@ eput.nhs.uk

\section{Copyright and usage}

(C) The Royal College of Psychiatrists 2018 
offence of 'wilful neglect', as introduced in the Criminal Justice and Courts Act 2015. At present, this offence, which carries a maximum jail term of 5 years, applies only to professionals who work in adult social care and health workers providing care for adults and children. Mr Cameron's proposals would also make it an offence for social workers, teachers and others not to report suspicions of current or past childhood sexual abuse. He stated:

'Professionals who fail to protect children will be held properly accountable and council bosses who preside over such catastrophic failure will not see rewards for that failure... Children were ignored, sometimes even blamed, and issues were swept under the carpet - often because of a warped and misguided sense of political correctness. That culture of denial which let them down so badly must be eradicated' (Department of Health 2015).

Legislation and the introduction of criminalising of non-disclosure, while well-intentioned, seek to address concretely what might be otherwise viewed as a complex set of dynamics related to abuse and exploitation. Denial is a core psychological feature of this, which needs to be understood as such and may not be amenable to 'eradication'. The wish for 'eradication' might be seen as akin to the legislative attempts to address substance misuse: although legislation can have a dramatic impact - as it has in banning smoking, a legal activity, in public illegal substance misuse has proven resistant to criminalisation as a deterrent.

\section{Current experience of universal mandatory reporting}

Mandatory reporting already exists in the USA, Canada and Australian states. The experience is mixed and has not had the desired effect of reducing child sexual abuse. In the USA since the introduction of mandatory reporting there has been a huge increase in reports over the decades, with a large number of unsubstantiated cases (78\%) (Tumlin and Green 2000; U.S. Department of Health and Human Services 2010), but the number of substantiated cases remains low. However, to counter this $74 \%$ of substantiated cases are identified by mandated reporters (U.S. Department of Health and Human Services 2010). In her presentation to the NSPCC round-table discussion, Professor Laura Hoyano stated that the results of mandatory reporting can overwhelm social services, causing them raise barriers to investigation and thus reducing the percentage of notifications that are investigated (NSPCC 2014). She also describes what she called the 'Pontius Pilate' effect, where mandatory reporting is seen as an intervention in itself: after a report has been submitted, it is assumed that 'experts' will
BOX 1 Risks of mandatory reporting

- Resources may be diverted into assessment and investigation rather than support of at risk individuals/children and families who then maybe less likely to be identified than before

- It does not guarantee effective child protection and creates a false sense of security

- Prosecutions for non-reporting are low, despite significant reported incidence of abuse

- It may deter victims from seeking help because of concerns about confidentiality

- Removing the autonomy of victims, especially children, to decide when to disclose abuse could have adverse consequences and lead to re-traumatisation

protect the child, and those surrounding the child relax their vigilance (Box 1).

Professor Ben Mathews reaches a different conclusion. He has written extensively about the Australian experience in a Royal Commission report into mandatory reporting (Mathews 2014a) and has also conducted a cross-jurisdictional comparison between the reporting and identification of severe child maltreatment in the Australian state of Victoria, where mandatory reporting exists, and Ireland, where it was only introduced in December 2017 (Mathews 2014b). In the latter Mathews makes a strong case for mandatory reporting as a response to 'a hidden phenomenon': his data analysis reveals that a substantially higher number of sexually abused children were identified in Victoria than in Ireland, with over half of the confirmed cases identified as a result of reports by mandated reporters. In his Royal Commission report he states that 'it is well-established that with only voluntary reporting, professionals including doctors, and others, are less likely to report, and this has a consequential adverse effect on the identification of cases of sexual abuse' (Mathews 2014a: p. 47) (Box 2).

BOX 2 The case for and aims of mandatory reporting of child sexual abuse

- Identifying cases of abuse that would otherwise remain hidden

- Preventing continuation of abuse of the child who is the subject of the report

- Detection of the offender

- Preventing further abuse by the offender Providing medical and other therapeutic help to the victim and their family 


\section{Ethical and clinical considerations}

Central to the conflict that doctors, psychiatrists and psychotherapists face with mandatory reporting is confidentiality. This conflict is captured in the General Medical Council (GMC) principle of confidentiality:

'Confidentiality is central to trust between doctors and patients. Without assurances about confidentiality, patients may be reluctant to seek medical attention or to give doctors the information they need in order to provide good care' (General Medical Council 2009: para. 6)

Without the assurance of confidentiality, victims and perpetrators of sexual abuse maybe reluctant to seek help for fear that disclosure would catapult them into the criminal justice system without their consent or proper preparation. At the same time their clinicians, in seeking to help and protect them from unwanted intrusion, would be impeded in their duty of care, and face being in a position of committing a criminal offence.

Disclosure is a process, not a one-off event, and it needs to be facilitated by a trusting professional relationship. Clinical experience and research undertaken by the NSPCC (Miller 2014) indicates that patients, and especially children, often test the responses of people involved in their care by partially disclosing what has happened to them and, as they gradually gain trust in the therapeutic relationship over time, building up to full disclosure.

The picture is similarly complicated when the reporting relates to adults' allegations of historical childhood sexual abuse. Although for some people, seeking help reporting to the authorities is part of their therapeutic journey, for others the need for confidentiality while they gradually explore their past experiences of abuse is essential to the process of disclosure and engagement in a therapeutic process. Any breach of the boundary of confidentiality is threatening to their engagement and sense of autonomy, and thus threatens to re-traumatise them.

\section{UK government consultation on mandatory reporting}

In 2016 the government launched a 12-week public consultation seeking views on both a mandatory duty to report and a duty to act, which would require certain practitioners and organisations to take appropriate action (which might include reporting) if they knew or had reasonable cause to suspect child abuse or neglect (HM Government 2016). The government's feedback on this consultation is still awaited. Organisations including the Royal College of Psychiatrists, NSPCC, National Association for People Abused in Childhood (NAPAC) and Specialist Treatment Organisation for the Prevention of Sexual Offending (StopSO) have published their responses. These vary from survivors in support of mandatory reporting to mixed responses such as that of the NSPCC, which supports limited mandatory reporting in closed institutions, for example boarding schools and care homes, and a duty to act that allows room for professional discretion. The Royal College of Psychiatrists cautiously favours the duty to act and strongly opposes mandatory reporting.

A case study in the College's feedback (Royal College of Psychiatrists 2016a) helpfully illustrates the dilemma that a clinician would face when confronted with a person with obsessive-compulsive disorder where the risk of harm appears high, but the actual risk is considered to be low. In this example the adult patient was plagued by an intrusive thought that she had committed an offence in her youth which in the present was difficult to distinguish from reality. It would have provided her some relief from her torment if her treating clinician had colluded with her internalised sense of guilt, compelled by a mandatory reporting duty to involve police rather than continuing to try to understand and work through the autobiographical context of her thought. This exploration and potential for help would have been foreclosed, and her well-being, professional and family life would all have been at risk. Other disorders could be also substituted here, for example people with psychotic beliefs of being subject to abuse or abusing others, where careful clinical consideration is required before action.

\section{Safeguarding}

Local authorities have a statutory role, under the Children Act 1989 and 2004, for safeguarding all children in their area (HM Government 2017) and safeguarding is also national priority for NHS England. Local safeguarding children boards (LSCBs) are responsible for local child protection policy and guidance, and coordinate effective working to protect children. Mandatory training in safeguarding is in place for all NHS staff and all NHS services have identified or designated healthcare professionals who provide expertise and ensure safeguarding training and fulfill other specialist safeguarding roles, including representation on LSCBs. They will also refer to the Local Authority Designated Officer (LADO) where there are allegations against staff members who work with children. The role of the LADO is to decide if there is a significant concern either to the child or a risk to others from the staff member. The Royal College of Psychiatrists advocates this current system (Royal College of Psychiatrists 2016b).

Mandatory reporting related to children under the age of 18 has existed for female genital mutilation 
(FGM) since March 2015. There are now clear pathways for involving the police when FGM is reported by a child or has been observed by a professional. This is helpful in the reporting of FGM, and figures have been available for the first time; these show that the vast majority of cases are performed outside the UK. However, there have been no successful prosecutions in the first year since the introduction of mandatory reporting or since FGM was made illegal in 1985. This suggests that there are complex sociocultural factors relating to the practice of FGM and that the impact of mandatory reporting is yet to be evaluated.

Mandating the reporting of current and historic child sexual abuse risks removing the capacity for clinical judgement for professionals in safeguarding roles and those seeking their advice.

\section{Conclusions}

There are compelling arguments both for and against mandatory reporting of child sexual abuse to improve child protection. Clinicians need to be able to act in their patients' best interests by maintaining confidentiality when necessary, but they must also protect others at risk. The bottom line is that as a society we need to do more to bring into the open what has previously been hidden, both to prevent abuse and to allow victims and perpetrators to access help in the knowledge that their privacy will be respected. This is a fine balance.

\section{References}

Chen L, Murad H, Paras M, et al (2010) Sexual abuse and lifetime diagnosis of psychiatric disorders: systematic review and meta-analysis. Mayo Clinic Proceedings, 618: 85.

Department of Health, Home Office, Ministry of Justice, et al (2015) PM unveils tough new measures to tackle child sexual exploitation (press release, 3 March). GOV.UK.

Felitti VJ, Anda RF, Nordenberg D, et al (1998) Relationship of childhood abuse and household dysfunction to many of the leading causes of death in adults: The Adverse Childhood Experiences (ACE) Study. American Journal of Preventive Medicine, 14: 245-58.

Flatley J (2016) Abuse during Childhood: Findings from the Crime Survey for England and Wales, year ending March 2016. Office for National Statistics.

General Medical Council (2009) Confidentiality. GMC.

Gray D, Watt P (2013) Giving Victims a Voice: Joint Report into the Sexual Allegations Made against Jimmy Savile. Metropolitan Police Service and NSPCC.

HM Government (2016) Reporting and Acting on Child Abuse and Neglect: Government Consultation. TSO (The Stationery Office).

HM Government (2017) Working Together to Safeguard Children: A Guide to Inter-Agency Working to Safeguard and Promote the Welfare of Children. TSO (The Stationery Office).

Mathews B (2014a) Mandatory Reporting Laws for Child Sexual Abuse in Australia: A Legislative History. Report for the Royal Commission into Institutional Responses to Child Sexual Abuse. Australian Centre for Health Law Research.

Mathews B (2014b) Mandatory reporting laws and identification of child abuse and neglect: consideration of differential maltreatment types, and a cross-jurisdictional analysis of child sexual abuse reports. Social Sciences, 3: 460-82.

Miller P, Allnock D (2014) No One Noticed, No One Heard: A Study of Disclosures of Childhood Abuse. NSPCC.

NSPCC (2014) Exploring the Case for Mandatory Reporting: A Summary of a Roundtable Hosted by the NSPCC. NSPCC.

Royal College of Psychiatrists (2016a) Consultation Response: Reporting and Acting on Child Abuse and Neglect. Royal College of Psychiatrists (https://www.rcpsych.ac.uk/pdf/RCPsych\%20consultation\%20response \%20and\%20supplementary\%20information.pdf).

Royal College of Psychiatrists (2016b) The Royal College of Psychiatrists Consultation Response. Home Office and Department for Education: 6.

Tumlin K, Green R (2000) The Decision to Investigate; understanding State Welfare Screening Policies and Practices: May. Washington, DC: Urban Institute.

U.S. Department of Health and Human Services (2010) Child Maltreatment 2009. Washington, D.C.: Administration for Children and Families.

Varese F, Smeets F, Drukker M, et al (2012) Childhood adversities increase the risk of psychosis: a meta-analysis of patient-control, prospective- and cross-sectional cohort studies. Schizophrenia Bulletin, 38 : $661-71$.

Zanarini MC, Williams AA, Lewis RE, et al (1997) Reported pathological childhood experiences associated with the development of borderline personality disorder. American Journal of Psychiatry, 154: 1101-6. 